\title{
Interferon lambdas: the next cytokine storm
}

\author{
Christabel Kelly, Paul Klenerman, Eleanor Barnes
}

Peter Medawar Building for Pathogen Research and Oxford NIHR Biomedical Research Centre, Nuffield Department of Medicine, University of Oxford, Oxford, UK

\section{Correspondence to} Dr Eleanor Barnes, Peter Medawar Building for Pathogen Research, Oxford NIHR Biomedical Research Centre, Nuffield Department of Medicine, University of Oxford, South Parks Road, Oxford OX1 3SY, UK;

ellie.barnes@ndm.ox.ac.uk

Published Online First 8 February 2011

\section{ABSTRACT}

For two decades the scientific community has sought to understand why some people clear hepatitis $C$ virus (HCV) and others do not. Recently, several large genome-wide association studies have identified single nucleotide polymorphisms (SNPS) linked to interferon lambda 3 (IFN $\lambda 3$ ) that are associated with the spontaneous resolution and successful treatment of HCV infection. These observations are generating intense research activity; the hope is that IFN $\lambda 3$ genetic variants may serve as important predictive biomarkers of treatment outcome and offer new insights into the biological pathways involved in viral control. A pharmacogenomic treatment approach for HCV can now be envisaged, with the incorporation of host genetic variants into a predictive treatment algorithm with other factors. The SNPs associated with the clinical outcome of HCV infection are located some distance from the IFN $\lambda 3$ gene itself, and causal genetic variants have yet to be clearly defined. Locating these causal variants, mapping in detail the IFN $\lambda 3$ signalling pathways and determining the downstream genetic signature so induced will clarify the role of IFN $\lambda 3$ in the pathogenesis of HCV. Clinical studies assessing safety and efficacy in the treatment of HCV with exogenous IFN $\lambda 3$ are currently underway. Early results suggest that IFN $\lambda 3$ treatment inhibits HCV replication and is associated with a limited side effect profile. However, hepatotoxicity in both healthy volunteers and HCV-infected patients has been described. This review discusses the genetic studies that link IFN $\lambda 3$ to both the spontaneous resolution and treatment-induced clearance of HCV and the potential impact of this in clinical practice, the biology of IFN $\lambda 3$ as currently understood and how this may impact on HCV infection, and describes the early studies that assess the role of this cytokine in the treatment of patients with HCV.

\section{INTRODUCTION}

For more than two decades, interferon- $\alpha$ (IFN $\alpha$ ) treatment has formed the cornerstone of therapy for hepatitis $\mathrm{C}$ virus ( $\mathrm{HCV}$ ) infection. Despite the identification of some viral and host factors associated with viral clearance, response to therapy remains highly unpredictable. Since treatment is prolonged, expensive and fraught with side effects, intense efforts have been made to identify biomarkers predictive of a successful treatment outcome. In 2009 there was a major breakthrough in this endeavour with the discovery that single nucleotide polymorphisms (SNPs) linked to the cytokine IFN $\lambda 3$ (also known as IL28B) are a dominant host factor at a population level in determining treatment outcome. Furthermore, the same SNPs are associated with the clinical outcome of primary infection-an intense area of research in the field of HCV over recent years. It is therefore not surprising that IFN $\lambda$ is creating a storm in the HCV arena.

This review describes the discovery of IFN $\lambda 3$ linked SNPs that are associated with HCV control, summarises the biology and function of IFN $\lambda 3$ as currently understood and describes the early studies that assess the role of this cytokine in the treatment of patients.

\section{Identification of IFN $\lambda 3$ as a key cytokine in HCV infection using genetic technology}

Over the last decade a number of host factors have been shown to play a role in the clinical outcome of HCV using either a candidate gene approach or through the assessment of biological pathways or demographic features that have an expected role in infection control. For example, in acute infection, particular human leucocyte antigens (both HLA classes I and II) and robust T cell immunity have been associated with viral clearance. ${ }^{1-3}$ Singlesource HCV outbreak studies most clearly demonstrate the importance of host factors in response to $\mathrm{HCV}$ infection. ${ }^{4}$ In these studies, young women were infected with the same inoculum of $\mathrm{HCV}$ from contaminated anti-D immunoglobulin. Approximately half of the women developed persistent infection while the remainder cleared the virus spontaneously, showing that host factors are important in determining clinical outcome. Similarly, in the treatment of persistent HCV infection, some host factors such as male gender and African ethnicity are associated with a poor treatment outcome. ${ }^{5-7}$ However, the actual predictive value of these factors is low, suggesting that other factors, hitherto unidentified, must exist. These observations provided the rationale for fully assessing the contribution of the host genome to the outcome of HCV infection.

The development of the human HapMap project in 2002 (http://hapmap.ncbi.nlm.nih.gov/) and advances in genetic techniques that facilitate the analysis of large datasets has led to an exponential growth in genome-wide association studies (GWAS). These studies associate common SNPs throughout the host genome with a disease cohort, and so identify important biological pathways in disease without prior knowledge or prejudice as to their relevance. This technique contrasts with candidate gene studies that take a pre-identified relevant gene and specifically assess its prevalence in the target population. The polarised clinical his paper is freely available online under the BMJ Jou gut.bmi.com/site/about/ unlocked.xhtml 
outcome of $\mathrm{HCV}$ infection (that of viral clearance versus viral persistence) makes $\mathrm{HCV}$ infection an ideal candidate for a GWAS approach.

The first GWAS in HCV infection was published by $\mathrm{Ge}$ and colleagues in September 2009 . $^{8}$ This study assessed the treatment outcome in a group of 1671 patients of mixed ethnicity (American European, Hispanic and African) receiving pegylated-IFN $\alpha$ (PEG- IFN $\alpha$ ) and ribavirin. A striking association was discovered between sustained viral response (SVR) with treatment and a cluster of seven SNPs linked to the IL28B gene, with the most significant SNP (rs12979860) demonstrating extremely high statistical significance $\left(p=1.06 \times 10^{-25}\right)$. No SNPs linked to other genes were associated with treatment outcome at genome-wide significance. Patients homozygous for the protective allele (C/C) had an SVR rate that was approximately three times higher than homozygotes for the risk allele (T/T) (78\% vs $28 \%$, and simply being a heterozygote carrier (T/C) for the risk allele also demonstrated approximately a twofold decrease in SVR (38\%) compared with those homozygous for the protective allele, suggesting a dominant role for the risk SNP. In assessing other factors linked to viral clearance, host IFN $\lambda 3$ genotype was more important than baseline viral load, the degree of liver fibrosis or ethnicity (figure 1).

Many other studies have since replicated these findings, demonstrating the robust association between IFN $\lambda 3$ and treatment outcome in other populations including Japanese, Australian, European, African American and Hispanic using either a GWAS approach ${ }^{8-11}$ or a candidate gene approach (table 1, figures 2 and 3). ${ }^{12-18}$

Shortly after the landmark publication by Ge et $a l^{8}$, the same gene (using a candidate gene approach assessing SNP 12979860) was shown to play a key role in the spontaneous resolution of disease following primary infection in both African and Caucasian populations. ${ }^{18}$ This study showed that carriers of the risk allele were three times less likely to spontaneously clear the virus than homozygotes for the protective allele. This finding was subsequently supported in a large GWAS by

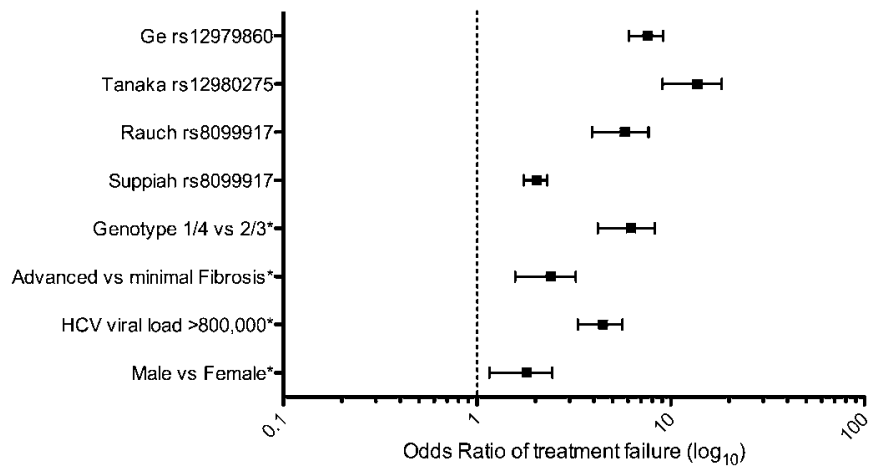

Figure 1 Odds ratio (OR) of treatment failure in hepatitis $C$ virus (HCV) infection. OR of risk factors associated with treatment failure including the interferon $\lambda 3$ (IFN $\lambda 3$ )-linked single nucleotide polymorphisms (rs8099917, rs12979860, rs12980275) in published genome-wide association studies. ${ }^{8-11}{ }^{*}$ Derived from Rauch et al. ${ }^{9}$

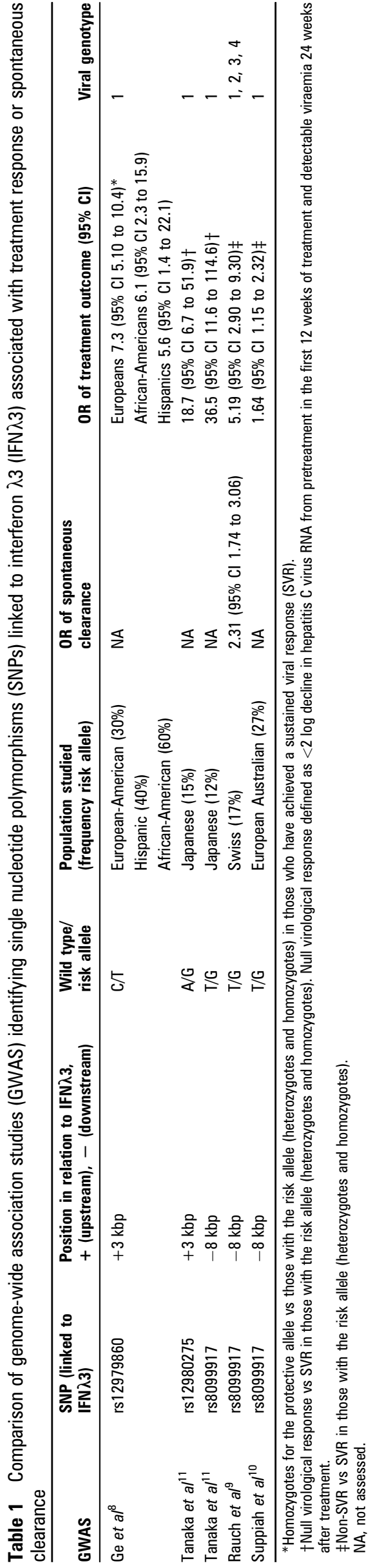

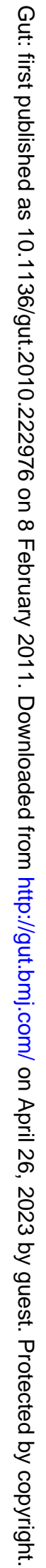




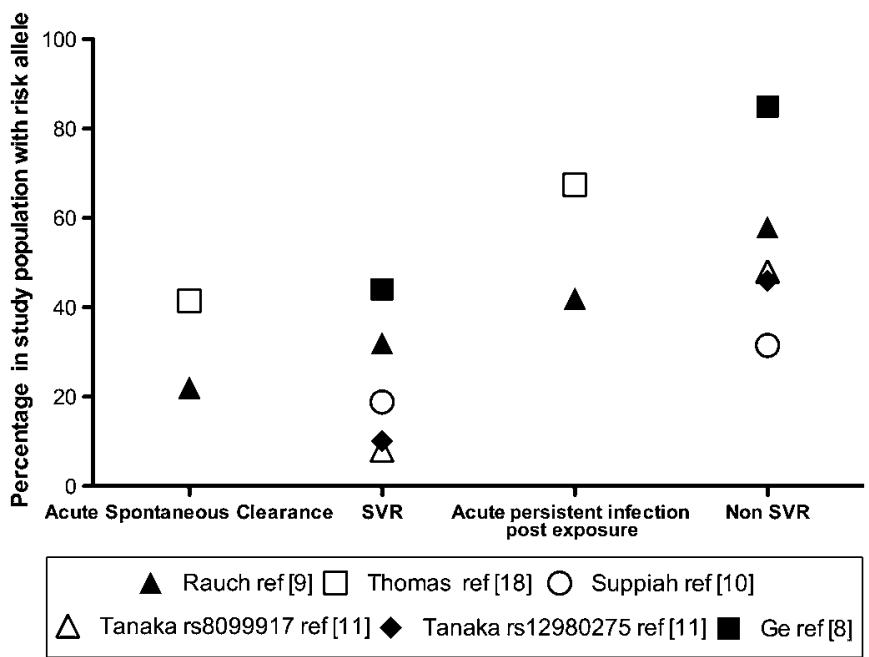

Figure 2 Interferon $\lambda 3$ (IFN $\lambda 3$ ) risk allele frequency in specific populations infected with hepatitis $C$ virus (HCV). Risk allele frequency in those who clear acute infection spontaneously and in those who have a successful treatment response (SVR) compared with individuals who develop persistent infection or who fail treatment (non-SVR). ${ }^{8-11} 18$

Rauch et al in both HCV-infected patients and HIV and HCV co-infected patients. It is now quite clear that the IFN $\lambda$ pathway plays a key role in natural host immunity as well as in the response to IFNa treatment of HCV infection.

Although the SNPs identified in the first phase of the GWAS above are linked to the IFN $\lambda 3$ gene, they are not located within known regulatory elements or the coding region of the IFN $\lambda 3$ gene itself. Rather, they have an expected association with 'functional' polymorphisms involved in the regulation or the function of the IFN $\lambda 3$ gene product. This is because IFN $\lambda 3$ has a distinct haplotype block (distinct regions of the host genome with SNPs that are in tight linkage disequilibrium with

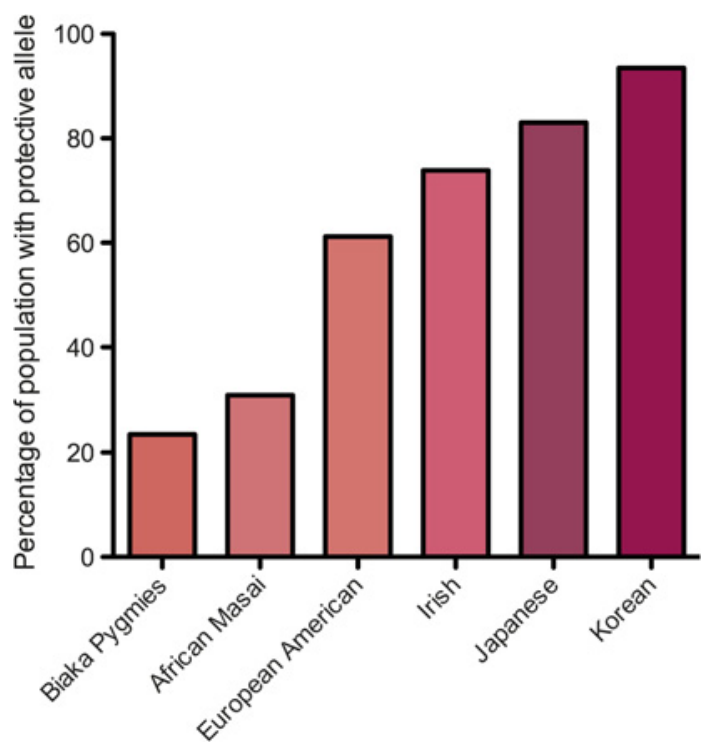

Figure 3 Mean frequency of interferon $\lambda 3$ (IFN $\lambda 3$ ) protective allele in different ethnic groups. The protective allele is less common in African populations than in Asian populations, correlating with the known poor outcome from hepatitis C virus (HCV) infection in patients of African origin. Figure derived from data in Thomas et al. ${ }^{18}$ one another) that extends beyond the gene and the gene regulatory elements. The most significantly associated SNP identified by Ge and colleagues (rs12979860) lies $3 \mathrm{~kb}$ upstream of the IFN 33 gene. ${ }^{8}$ In an attempt to identify the functional (or causal) polymorphism, these authors sequenced the IFN $\lambda 3$ gene itself in 96 individuals and found two variants (one 37 base pairs upstream of the translation initiation codon (rs28416813) and a non-synonymous coding SNP (rs8103142) encoding an amino acid substitution within the IFN $\lambda 3$ gene. However, these variants were so tightly linked that further genotyping of the entire cohort failed to show that any of these SNPs were uniquely linked to treatment outcome. Several studies have identified SNP rs8099917 as that most significantly associated with outcome. ${ }^{9-11}$ This SNP is located $8.9 \mathrm{~kb}$ from the end of transcription of IFN $\lambda 3$ in the intergenic region between IFN 22 and IFN 3 . Suppiah and colleagues further clarified the association and genotyped $20 \mathrm{SNPs}$ in the IFN $\lambda 3$, IFN $\lambda 2$, IFN $\lambda 1$ gene cluster region. ${ }^{10}$ Haplotype analysis identified a six-allele haplotype that was tagged by rs809917 (rs12980275, rs8105790, rs8103142, rs10853727, rs8109886 and rs8099917) linked to SVR. Notably, this haplotype encompasses regulatory elements from both IFN $\lambda 3$ and IFN $\lambda 2$. Tanaka and colleagues also sequenced 48 patients and established a similar seven-allele haplotype (rs8105790, rs11881222, rs8103142, rs28416813, rs4803219, rs8099917 and rs7248668) associated with SVR (OR 11.1 $\left(\mathrm{p}=1.35 \times 10^{-25}, 95 \%\right.$ CI 6.6 to 18.6$) .{ }^{11}$ Similarly, Rauch and colleagues, in searching for the causal variants, resequenced the IL28B locus in 47 individuals with and without the SNP rs8099917 and with different treatment outcomes and identified 21 SNPs, of which five tightly linked SNPs were thought to be potentially causative. ${ }^{9}$

Since multiple tightly-linked SNPs within and around the IFN $\lambda 3$ gene are associated with SVR, it seems unlikely that sequence studies of the IFN $\lambda 3$ region in association with known clinical outcome will identify which of these are causal. In vitro IFN $\lambda 3$ functional assays combined with mutagenesis studies will determine the effect of these polymorphisms on IFN $\lambda 3$ function, although even these may not prove causality. A further note of caution: while it is generally assumed that common variants associated with disease in GWAS reflect the effect of a causal variant in close proximity, this is not necessarily so. Rare causal variants cannot be detected using GWAS technology, which only screens the human genome for common SNPs. An alternative possibility, therefore, is that multiple rare variants that lie distant from the common variant identified in GWAS occur stochastically and more frequently in association with the GWAS common variant. These associations have been termed 'synthetic'. ${ }^{19}$ In this scenario a common variant identified in the GWAS will be erroneously linked to a disease due to its chance association with the true causal variants which lie undetected by GWAS methodology. This possibility has been demonstrated by the analysis of existing datasets 
showing that known rare causal mutations responsible for sickle cell disease and hearing loss can give rise to genome-wide significant synthetic associations that lie a considerable distance $(2.5 \mathrm{Mb})$ from the causal mutations. ${ }^{20}$ These observations do not detract from the use of the GWAS variants in predicting treatment outcome in HCV infection, but they do raise the possibility that the biological mechanisms that underpin this are related to distant multiple rare genetic variants.

It has been known for some time that ethnicity affects treatment outcome of HCV infection, with viral clearance rates lowest in African-Americans, highest in East Asians and intermediate in European-Americans. ${ }^{5-7}$ We now know that the underlying rate of carriage of the IFN $\lambda 3$ risk allele depends on the ethnic group of the population under study (figure 3). This suggests that ethnic differences in the IFN $\lambda 3$ gene may explain, at least in part, the different response rates to therapy in different ethnic groups. Although one study did not find an association with IFN $\lambda 3$ in the AfricanAmericans studied, it is likely the group $(n=53)$ was underpowered to identify the association. ${ }^{15}$

The data supporting a major role for the IFN $\lambda 3$ gene in clinical outcome is less clear for genotypes 2 and 3 infection than for genotype 1. The largest study of 1362 patients by Rauch and colleagues ${ }^{9}$ and a smaller Spanish study ${ }^{14}$ found no association between genotypes 2 and 3 and IFN $\lambda 3$ polymorphisms, although the treatment failure rate in both studies was very low (37/230 patients and $9 / 59$ patients, respectively). In contrast, two other groups have found that IFN $\lambda 3$ status can predict SVR in genotypes 2 and 3 infection, although in one of these studies the effect was restricted to patients who did not achieve a rapid virological response. $^{13} 15$ Intriguingly, McCarthy and colleagues also showed that the protective IFN $\lambda 3$ genotype is enriched in patients infected with genotype $3 \mathrm{HCV}$ compared with genotype 1 $(p=0.0007) .{ }^{15}$ The mechanism behind this observation is speculative but, since the protective allele is almost universal in Asian populations where genotype 3 infection has evolved, ${ }^{18}$ it seems plausible that this genotype may have evolved mechanisms to persist following acute infection in the face of the protective allele.

It remains to be seen how we can best use the information about IFN $\lambda 3$ in clinical practice. In genotype 1 infection, IFN $\lambda 3$ is the best predictor of treatment outcome when compared with other known baseline predictors including fibrosis score, viral load, gender and ethnicity. 891115 However, the sensitivity, specificity and predictive value of the IFN $\lambda 3$ genetic variants currently identified are too low to be clinically useful alone. It has been calculated that the risk allele predicts non-response to treatment with a sensitivity of $57 \%$ and specificity of $63 \%$ with a positive predictive value of $64 \% .{ }^{10}$ For homozygotes with the protective allele, SVR can be predicted with $65 \%$ sensitivity and $78 \%$ specificity in patients with genotype $1 .^{15}$ It is plausible, however, that IFN $\lambda 3$ alleic status will in future be incorporated into a treatment algorithm with other known predictors.

\section{THE BIOLOGY OF INTERFERON LAMBDAS}

The GWAS point to IFN $\lambda$ as a key cytokine in the control of HCV infection. Is this biologically plausible? What do we know currently about the role and function of IFN $\lambda$ ?

Since their discovery in $2003,{ }^{21}{ }^{22}$ IFN $\lambda$ s (a type III IFN) have largely been considered a 'poor relative' of type I IFNs. Many functions appeared to overlap with type I IFNs and until now there has been little evidence of an independent and clinically significant role. Although the GWAS data suggest that IFN $\lambda 3$ may be the type III IFN most relevant to the pathogenesis of $\mathrm{HCV}$, historically the assessment of IFN $\lambda$ biology has focused on IFN $\lambda 1$ and IFN $\lambda 2$.

\section{Classification}

IFN $\lambda$ s are classified within the class II cytokine family based on the similarity of their receptors. The class II family of cytokines consists of three types of IFNs (types I, II and III) as well as the interleukin 10 (IL-10)-related cytokines. Type I IFNs include IFN $\alpha$ and IFN $\beta$, type II IFN is IFN $\gamma$ and type III IFNs consist of IFN $\lambda 1$, IFN $\lambda 2$ and IFN $\lambda 3$ which are also called IL29, IL28A and IL28B, respectively. IFN $\lambda 3$ shares considerable amino acid sequence homology with IFN $22(96 \%)$ but less with IFN $\lambda 1$ (81\%). ${ }^{21}$ There are currently very limited available data comparing the different biological activity of the three different $\lambda$ cytokines.

Although class II cytokines may be quite divergent at the amino acid level, they are structurally related with a shared $\alpha$-helical pattern and signal through related heterodimeric transmembrane protein receptors. However, biological activity ultimately depends on the receptor cytoplasmic domains, which are not related, and these may trigger overlapping but different biological functions. This, in addition to the pattern of receptor distribution among different cell types, means that the different type II cytokines are functionally distinct.

\section{Production of IFN $\lambda$}

Broadly speaking, the IFN $\lambda$ family is similar to IFN $\alpha$ in that it has both antiviral and immunomodulatory properties, but there are important differences. Whereas IFN $\alpha$ is produced by all nucleated cells, IFN $\lambda$ may be produced by fewer cell types. $^{23}$ Plasmacytoid monocyte-derived dendritic cells ( $\mathrm{pDC}$ and MDDC) and macrophages produce IFN $\lambda$ in response to influenza virus infection and/ or to bacterial and viral molecular mimics (the Toll-like receptor agonists lipopolysaccharide and poly I:C). ${ }^{24} 25$ Interestingly, macrophage production of IFN $\lambda$ is markedly enhanced by pre-culturing these cells with IFN $\alpha$-evidence of interplay between the two pathways. ${ }^{26} 27$ There is evidence that IFN $\lambda$ secretion may be influenced by cytokines-for example, pDCs produce an 


\section{IFN $\lambda 3$ and genetic technology}

SNPs are single base pair mutations and are the main sites of genetic variation between individuals.

- SNPs linked to the IFN $\lambda 3$ gene predict both treatment response to PEG-IFN $\alpha$ / ribavirin and spontaneous clearance of HCV.

- Individuals either heterozygous or homozygous for the risk allele are less likely to clear HCV infection than those homozygous for the protective allele.

- African populations have a higher frequency of the risk allele than Caucasian or East Asian populations.

- Carriers of the IFN $\lambda 3$ risk allele are 2-12 times more likely to fail treatment than homozygotes for the protective allele.

- The predictive value of the IFN $\lambda 3$ alleles alone is insufficient to determine treatment outcome but may be a useful component of a treatment algorithm in the future.
IFNs that are not fully understood. All class II cytokines appear to activate STAT1 and STAT3 and bind to IFN $\gamma$-activated sites (GAS) to various extents in the cell nucleus, resulting in the transcription of IFN-stimulated genes (ISGs). However, in addition, type I IFNs and IFN $\lambda$ also activate IFNstimulated response elements (ISRE) through the activation of STAT2 in association with STAT1 and IRF9 and which again results in the production of IFN-stimulated genes (figure 4). ${ }^{33} 34$

\section{Biological effects of IFN $\lambda$}

Microarray experiments have shown that hundreds of ISGs are produced in response to IFN stimulation. The full spectrum of genes that are stimulated in response to different IFNs has not been defined, but it is clear that even different IFNs that bind to the same receptor may ultimately induce different genes ${ }^{3536}$ and that mutations in a single amino acid in key locations in the IFN $\lambda$ binding site can have a significant impact on antiviral activity, altering antiviral activity by up to 300 -fold. ${ }^{37}$ Marcello et al evaluated ISG expression, protein production and HCV RNA replication using the $\mathrm{HCV}$ replicon system with cell culture HCV in response to IFNa and IFN $\lambda$ stimulation. ${ }^{38}$ Both cytokines ultimately inhibited HCV replication in this system. However, the kinetics of STAT activation and the potential effector genes induced differed. In particular, IFNa genes peaked and declined rapidly whereas IFN $\lambda$ genes increased steadily. Thus, the functional differences and the interplay between types I and III IFNs are likely to lie in the fine detail of this signalling pathway, yet to be fully elucidated for IFN $\lambda$, and in the differential distribution of cells that both produce IFN $\lambda$ and are able to respond to IFN $\lambda$ through the presence of appropriate receptors.

The effects of IFN $\lambda$, like that of IFN $\alpha$, on immune cell function appear to be complex and diverse. This means that in vitro studies of discrete functions, such as the in vitro effects of IFN $\lambda$ on an isolated cell type, may tell us little about the in vivo effects in a particular disease setting where a complex interplay of cell types and inflammatory stimuli is at work. With that proviso, IFN $\lambda$ has been shown to decrease the production of Th2-type cytokines (IL-4, IL-5, IL-13, IL-14, IL-15), potentially favouring a Th1 immune pathway, ${ }^{39-41}$ increase $\mathrm{T}$ regulatory cells ${ }^{32}$ and augment CD8 T cell cytotoxicity and memory responses in macaques vaccinated with an HIV antigen. ${ }^{42}$ However, others have shown that immune cell subsets (monocytes, NK, T cells) are unresponsive to IFN $\lambda 1$ and IFN $\lambda 2$ and postulated that this is due to the production of a soluble receptor produced by peripheral blood mononuclear cells. ${ }^{30} 3243$

The antiviral effects of IFN $\lambda$ are clearer; in vitro IFN $\lambda$ protects human cell lines against the cytopathic effects of vesicular stomatis virus (VSV) and encephalomyocarditis virus ${ }^{21} 22$ and, in hepatocyte models, IFN $\lambda 1$ inhibits both HCV and HBV replication. ${ }^{31} 3844$ However, some studies have shown that, unlike IFN $\alpha$, IFN $\lambda$ is unable to suppress $\mathrm{HCV}$ completely $^{38}$ and all groups found it was less 

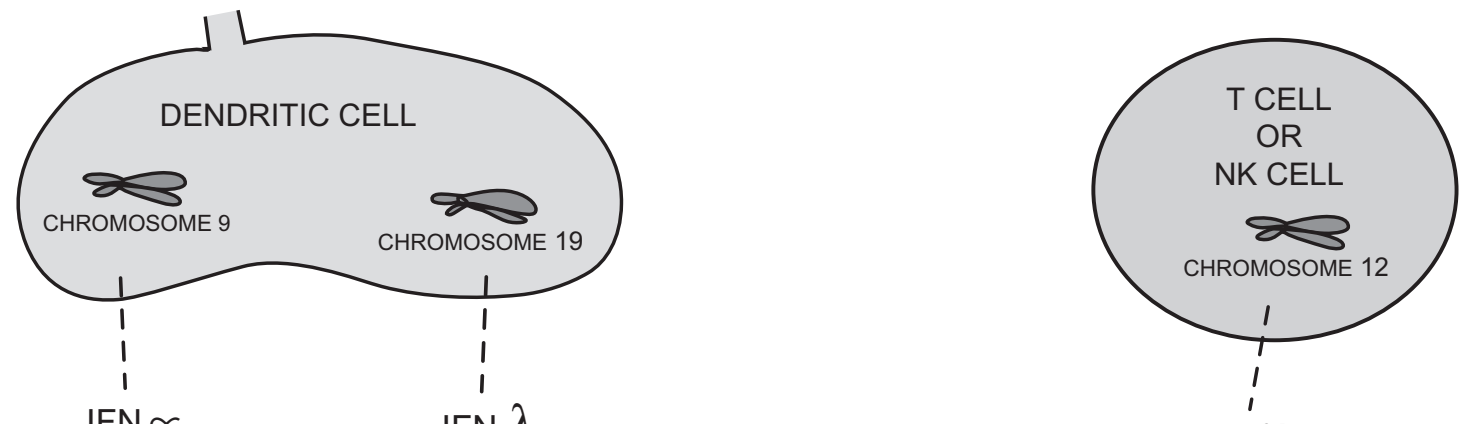

IFN $\lambda$

IFN $\gamma$
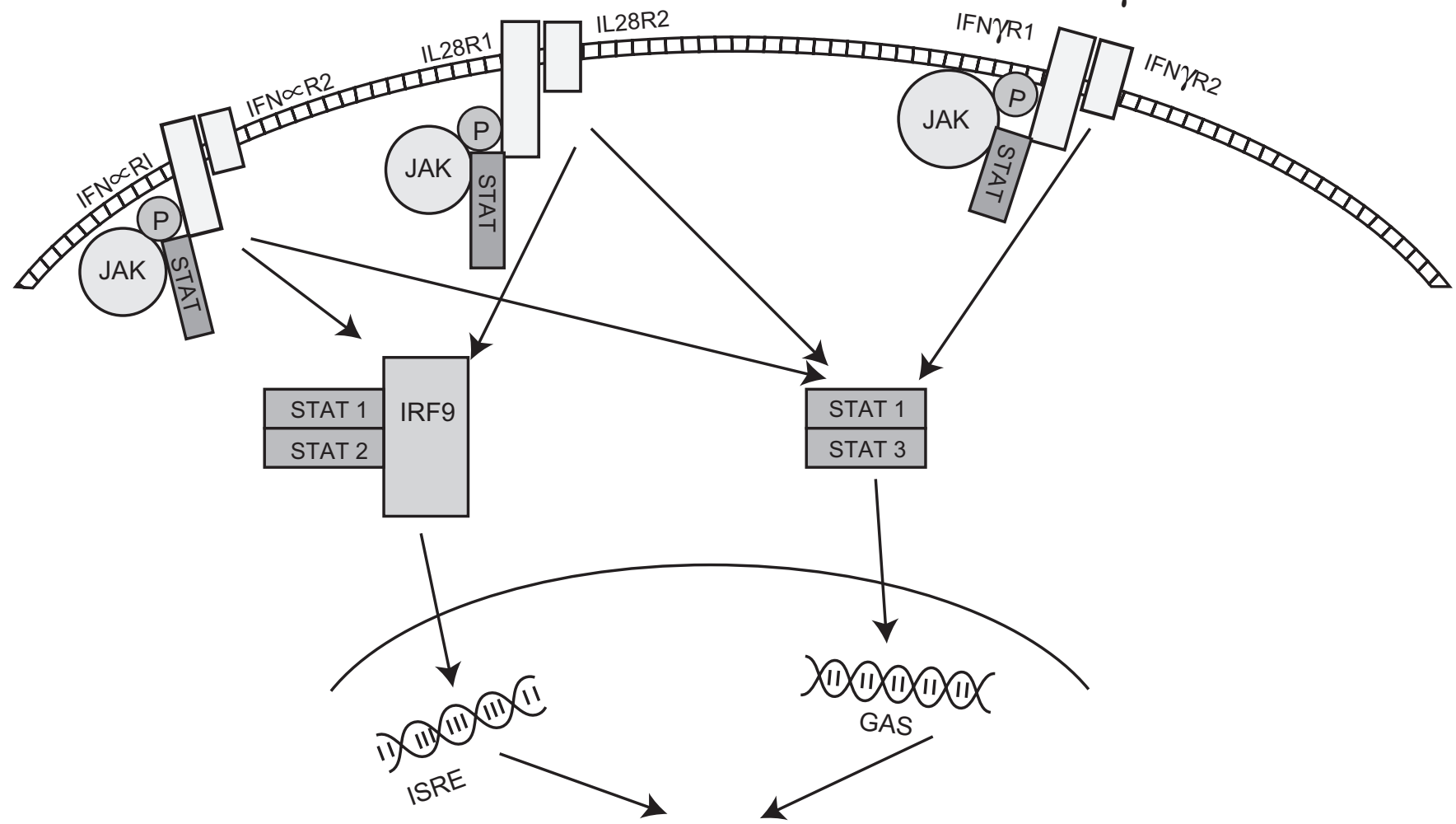

IFN stimulated

gene transcription

Figure 4 The signalling pathway of type III interferons (IFNs) compared with type II and type I IFNs. IFN $\lambda$ binds the IFN $\lambda$ R1 chain leading to a conformational change in the receptor. It recruits a second short receptor chain (IL10R2). JAK 1 and Tyk 2 transphosphorylate the receptor chains and form phosphorylated tyrosine peptides on the IFN $\lambda$ R1 receptor chain. STAT proteins bind and are phosphorylated. They can then form homo- or heterodimers and migrate to the nucleus to bind gene regulatory elements (gamma-activated sequences, GAS). In the case of type I and type III IFNs, they can also bind interferon regulatory factor 9 (IRF9) in the cytoplasm and then migrate to the nucleus to bind interferon stimulated regulatory elements (ISREs) to regulate gene transcription.

potent than IFN $\alpha$. The antiviral effects of IFN $\lambda$ on $\mathrm{HCV}$ infection in patients have also been demonstrated and are discussed further below. ${ }^{45}$ In this case, however, it is unclear if the antiviral effects are mediated directly or through the stimulation of immune cells, or both. It should be remembered that, in the case of chronic HCV infection, the presumed effects of IFN $\lambda$ on viral clearance revealed in the GWAS are mediated through treatment with exogenous IFN $\alpha$. How then might these cytokines interact with one another? Although IFN $\lambda$ may be less potent than IFN $\alpha$ as a direct antiviral, together these cytokines may have an additive effect. ${ }^{27} 3846$ In macrophages and hepatoma cell lines it has been shown that IFN $\alpha$ upregulates IFN $\lambda 1$ production 1000-fold and IFN $\lambda 2 / 310-100-$ fold. ${ }^{26} 27$

\section{Effect of IFN $\lambda 3$ polymorphism on IFN $\lambda$ biology}

It is not yet clear how-or indeed if-IFN $\lambda 3$ polymorphisms linked to the risk allele identified in the GWAS affect immune function or exert specific antiviral effects in HCV-infected patients. This will be a difficult task unless a causal IFN $\lambda 3$ variant is identified, enabling the precise assessment of the relevant IFN $\lambda 3$ variants in vitro. Currently, this assessment can only be made indirectly by observing immune function or HCV viral loads in people with and without the risk allele that lies a considerable distance from the IFN $\lambda 3$ gene. It is currently not clear if IFN $\lambda 3$ production by peripheral blood mononuclear cells (PBMCs) is associated with the IFN $\lambda 3$-linked genetic variants; while two studies found that the protective allele is associated 


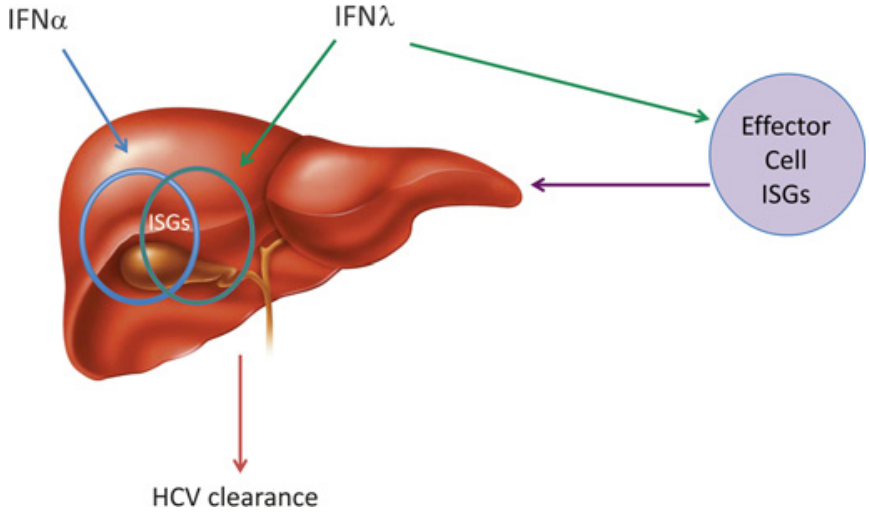

Figure 5 Model of the possible action of interferon $\lambda$ (IFN $\lambda$ ) in chronic hepatitis $C$ virus (HCV) infection. In chronic HCV infection, IFN $\alpha$ upregulates hepatic interferon-stimulated genes (ISGs) (represented by blue circle) but this fails to clear HCV. Addition of IFN $\lambda$ may result in stimulation of different ISGs (represented by green circle) and therefore HCV clearance. The IFN $\lambda$ polymorphism could impact on the expression of the right hepatic ISGs directly or through the stimulation of effector cell ISGs resulting in HCV clearance.

with increased levels of IFN $\lambda 3$ in PBMCs, ${ }^{10} 11$ others have found it to be unrelated. ${ }^{8}$ However, a consistent finding appears to be that patients with the IFN $\lambda$ 3-linked protective allele have a higher pretreatment HCV viral load than those with the risk allele, with $\mathrm{Ge}$ and colleagues finding that those homozygous for the protective allele had a viral load of $6.35 \log _{10} \mathrm{IU} / \mathrm{ml}$ compared with 6.16 $\log _{10} \mathrm{IU} / \mathrm{ml}$ in those homozygous for the risk allele $\left(p=1.21 \times 10^{-10}\right) .^{8} \quad 15$ This is paradoxical since a higher pretreatment viral load has been associated with a poor response to subsequent IFN $\alpha$ treatment. It should be noted, however, that the presence or absence of the risk allele does not associate with the clinically relevant viral load cut-off $\left(5.78 \log _{10} \mathrm{IU} / \mathrm{ml}\right)^{47} 48$ that has been associated with response to IFN $\alpha$ therapy, and it is therefore likely that the effect of the protective allele on clinical outcome is independent of the effect of this allele on viral load.

IFN $\alpha$ and IFN $\lambda$ ultimately exert their functions through the upregulation of ISGs. However, it has been repeatedly observed that a poor response to exogenous IFN $\alpha$ treatment is associated with a higher intrahepatic ISG expression before treatment. ${ }^{49}{ }^{50}$ One plausible explanation is that, in this setting, the IFN $\alpha$ pathway (and thus ISG expression) is already maximally stimulated and is therefore unresponsive to further exogenous IFN $\alpha$ treatment. A recent study has shown that the protective allele identified in the GWAS (rs8099917) does not correlate with intrahepatic IFN 33 production. ${ }^{49}$ Technically there are reasons why this might be so; for example, the low levels of IFN $\lambda 3$ detected in general make correlations difficult and by the fact that, owing to sequence homology between IFN $\lambda 3$ and IFN 22 , quantification by real-time PCR does not distinguish between these cytokines. However, the protective allele does correlate with a lower expression of ISGs and, in those with the risk allele, lower levels of intrahepatic IFN $\lambda 3$ were associated with higher ISG (Mx1 IFI44 and IFIT1) expression. ${ }^{49}$ In fact, the assessment of ISGs in response to IFNs is not straightforward given that many hundreds of these are expressed in liver tissue that is composed of hepatocytes and also of immune and other cell types. For example, in the study by Honda and colleagues, 1359 genes were differentially expressed between individuals with and without the protective allele. Pathway analysis suggested that IFN action and apoptosis were upregulated in the liver of those with the risk allele, whereas immune cell (B, NK and dendritic cells) genes were expressed in those with the protective allele. ${ }^{49}$ This is supported by the observation that ISG expression is upregulated in Kupffer cells (liver macrophages) of IFNa responders compared with non-responders, whereas ISGs are upregulated in hepatocytes of non-responders compared with responders. ${ }^{51}$ In other words, the protective effect of the IFN $\lambda 3$ allele may relate to the effects on or by 'visiting' cell populations rather than hepatocytes themselves.

In the setting of acute HCV infection, assuming the GWAS have correctly identified a genetic signal linked to IFN $\lambda 3$, protective IFN $\lambda 3$ variants could mediate viral clearance through immune stimulation or direct antiviral effects through the stimulation of the 'right' ISGs. Exactly what these ISGs are and in which cells they are produced is not yet clear. In the setting of chronic infection where IFN $\lambda 3$ variants also appear to be associated with response to exogenous IFN $\alpha$, the protective IFN $\lambda 3$ variant may well be linked to a complex intrahepatic ISG profile consisting of the 'right' ISGs produced by particular cell types (figure 5). Undoubtedly, identifying exactly what the right ISGs are is a challenging but key question that will be addressed in months to come.

\section{CLINICAL STUDIES}

IFN $\lambda$ is potentially an attractive alternative to IFN $\alpha$ for the treatment of $\mathrm{HCV}$ infection. IFN $\alpha$ has been used to treat HCV since the discovery of the virus two decades ago. Over this time, important modifications to IFN $\alpha$ - such as the addition of polyethylene glycol (PEG) to increase drug half-life and the addition of ribavirin to treatment regimes-have been incorporated. Although in the coming years HCV protease and polymerase therapies will add to the anti-HCV drug armoury, the rapid emergence of HCV variants which are resistant to these new drugs means that IFN $\alpha$ will probably remain an essential component of treatment regimens. However, treatment with IFN $\alpha$ is associated with a very significant adverse event profile. Thrombocytopenia, neutropenia and psychiatric complications such as anxiety, depression and sleep disturbance are particularly troubling. An IFN with potent antiviral effects and a more favourable side effect profile would revolutionise the treatment of HCV. In vitro experiments have shown that that IFN $\lambda$ has a limited effect on CNS and haematopoietic cells. So, with its promising antiviral activity in the liver but restricted receptor distribution, IFN $\lambda$ seems like the ideal candidate for development into a therapeutic agent. 


\section{The biology of IFN lambdas}

IFN $\lambda$ is a type III IFN of which there are three types: IFN $\lambda 1$, IFN $\lambda 2$, IFN $\lambda 3$ (IL29, IL28A, IL28B).

- Plasmacytoid dendritic cells are the main producers of IFN $\lambda$ stimulated by viruses and viral or bacterial mimics.

- IFN $\lambda$ has a restricted receptor distribution and therefore restricted targets.

- INF $\lambda$ has antiviral, antitumour and immunomodulatory effects.

- Upregulated hepatic ISGs pretreatment are associated with the IFN $\lambda 3$ risk allele and with poor treatment outcome.

- The effect of IFN $\lambda 3$ on HCV clinical outcome is likely to be a complex interplay between IFN $\alpha$, the virus itself, ISGs and immunomodulation.

Since the biological potential of IFN $\lambda$ s in general was recognised before the studies that implicated IFN $\lambda 3$ in particular, clinical trials to date have not focused on IFN $\lambda 3$ but rather on IFN $\lambda 1$. Zymogenetics (in collaboration with Bristol-Myers Squibb) are assessing PEG-IFN $\lambda 1$ in clinical studies which, while showing promising antiviral activity, have also uncovered a different but nevertheless significant side effect profile from that seen with IFNa. A phase I dose escalation study of PEGIFN $\lambda 1$ was presented in 2007 as an abstract (http://www.zymogenetics.com/products/docum ents/HEP_DART_PEG_IFN_12-07.pdf). Volunteers received a single subcutaneous injection of PEG-IFN $\lambda 1$. The study was stopped after volunteers received $7.5 \mu \mathrm{g} / \mathrm{kg}$, below the planned target maximum of $40 \mu \mathrm{g} / \mathrm{kg}$, after four volunteers developed significantly elevated transaminases. As there was evidence of bioactivity at lower doses that were not associated with hepatotoxicity, and since peak serum concentrations at tolerated doses were higher than those shown to have antiviral effect in vitro, ${ }^{31} 38$ a phase I study in HCV-infected patients followed.

In this 4-week open-label trial, patients with genotype-1 HCV infection received Peg-IFN $\lambda 1$ weekly or two-weekly. ${ }^{45}$ The study included both treatment-naïve patients $(n=7)$ and patients with previous virological relapse with standard therapy $(n=49)$. Despite the lower doses in this trial $(0.5-3 \mu \mathrm{g} / \mathrm{kg})$, six patients stopped or had treatment withheld due to elevated transaminase levels and four patients had raised lipase or amylase elevations which resolved after stopping PEGIFN $\lambda 1$. Although adverse effects were more

\section{Clinical studies}

- A phase I trial of PEG-IFN $\lambda 1$ has shown promising antiviral activity in both treatment relapsers and treatment-naïve patients with HCV.

- Hepatotoxicity has been associated with the clinical use of PEG-IFN $\lambda 1$.

- Impaired IFN $\lambda$ production may lead to increased susceptibility to viral infections.

- IFN $\lambda 3$ provides the opportunity to apply pharmacogenomics to HCV treatment. common in the highest dose group, it is noteworthy that, at only $1.5 \mu \mathrm{g} / \mathrm{kg}$, one patient developed severe hepatotoxicity with alanine transaminase levels of $667 \mathrm{IU} / \mathrm{ml}$ and bilirubin levels of $348.9 \mu \mathrm{mol} / 1$. Furthermore, one patient developed idiopathic thrombocytopenic purpura and three patients developed antibodies to IFN $\lambda 1$ during this short treatment course. Two patients previously treated with IFN $\alpha$ had cross-reactive antibodies to IFN $\lambda$ at baseline (although only one of these were neutralising antibodies), suggesting that previous treatment with IFN $\alpha$ may limit IFN $\lambda$ efficacy in some cases.

In keeping with the in vitro data showing a limited IFN $\lambda$ receptor distribution, ${ }^{23} 30$ there was a noticeable decrease in IFN $\alpha$-type side effects. Fatigue was the most common side effect $(28.6 \%)$ followed by nausea and myalgia $(12.5 \%$ and $10.7 \%$, respectively). However, insomnia and influenza-like illness were only experienced in $5.4 \%$, well below rates reported with IFN $\alpha$ and significant neutropenia did not occur. This trial also showed that IFN $\lambda 1$ is capable of antiviral activity in vivo. In patients receiving a dose of $\geq 1.5 \mu \mathrm{g} / \mathrm{kg} /$ week, $23 /$ 24 patients with treatment relapse achieved a fall in viral load of $\geq 2 \log _{10}$ and four of these achieved a rapid virological response, defined as undetectable viraemia at 4 weeks. Interestingly, all eight AfricanAmerican patients (a group known to respond poorly to IFN $\alpha$ treatment) in this study had a decline in viral load of $>2 \log _{10}$ at the end of treatment. The association of virological response to PEG-IFN $\lambda$ and IFN $\lambda$ genotype has very recently been assessed in an ongoing phase II study (published in abstract form only) of PEG-IFN $\lambda 1$ or PEG-IFN $\alpha$ with ribavirin combination therapy for 24-48 weeks, with a 2-week lead-in period of PEG-IFN $\lambda 1$ or PEG-IFN $\alpha$ alone, in patients with genotypes $1-4 \mathrm{HCV}$ infection. ${ }^{52}$ Interim results from patients receiving $120-240 \mu \mathrm{g}$ PEG-IFN $\lambda 1$, revealed a $71 \%$ viral response (VR) ( $\geq 2 \log _{10}$ reduction in viral load) at week 4 in those with a favourable genotype compared with $25 \%$ VR in those carrying the risk allele. ${ }^{52}$ In this study, grade $2 / 3$ hepatotoxicity was again observed (alanine transaminase or aspartate aminotransferase 2.5-20 times upper limited of normal) in $20 \%$ of patients on PEG-IFN $\lambda 1$ which was, however, successfully managed with dose reduction. ${ }^{52}$

IFN $\lambda$ may also in the future be considered both in the context of cancer therapy since this cytokine has been shown to suppress tumorigenesis in vitro $^{27} 3138$ and also as an immunomodulatory agent in the suppression of Th2-associated diseases such as allergy and atopy. ${ }^{39-41}$ IFN $\lambda$ may have a particular role in asthma since it suppresses IL-13 (known to be elevated in patients with asthma), ${ }^{39}$ and deficient IFN $\lambda$ production in the bronchi of patients with asthma is associated with an increase in respiratory infections in this population. ${ }^{53}$

The real utility of PEG-IFN $\lambda 1$ in the treatment of $\mathrm{HCV}$ will become more apparent following the final results from the ongoing phase II trial described above which will assess both safety and 
efficacy. ${ }^{52}$ Ultimately, new agents for HCV will need to be tolerable in the setting of pre-existing liver inflammation and poor hepatic reserve associated with liver cirrhosis.

\section{CONCLUSION}

The future is likely to bring IFN $\lambda 3$ and this exciting cytokine family further into the limelight, with head-to-head clinical trials between PEG-IFN $\lambda 1$ and PEG-IFN $\alpha$ underway for both HCV and HBV. Importantly, although genetic studies have now suggested that IFN $\lambda 3$ has a pivotal role in HCV clinical outcome, most work to date has been with IFN $\lambda 1$. IFN $\lambda 3$ may yet be developed as an alternative HCV treatment, particularly in those with the risk polymorphism, and genotyping for IFN $\lambda 3$ related SNPs may become part of a treatment decision algorithm. The differential effect of IFN $\lambda$ treatment on patients with and without the IL28B polymorphism is clearly an area worth further evaluation. Pharmacogenomic treatment approaches are one of the future challenges of medicine, and HCV provides the ideal opportunity to tailor potentially toxic therapies to individuals most likely to benefit. Whether work on this cytokine is applicable to genotypes other than 1 is still an open question, as is establishing the ideal therapeutic window for treatment with type III IFNs which have demonstrated hepatotoxicity in both healthy volunteers and patients with HCV infection. Furthermore, elucidating the pathway by which the IFN $\lambda 3$ polymorphism affects the response to acute infection and to IFN therapies remains an important future challenge. It is likely that defining the pattern of gene stimulation by IFN $\lambda$ in different cell types, as well as potential structural differences in IFN $\lambda 3$, will be critical in solving this puzzle. In particular, investigating the immunomodulatory effect of IFN $\lambda$ may well be key to unlocking the mechanism behind both its antiviral effect and its toxicities. Overall, the discovery of the link between the IFN $\lambda 3$ locus and HCV disease outcome has been a huge step forward for the field. However, while it has provided momentum for clinicians and basic scientists alike, the actual route ahead still needs careful charting. Nevertheless, while the destination is not yet clear, the journey itself will certainly be interesting.

Funding CK is funded by the Oxford NIHR Biomedical Research Centre and Wellcome Trust UK, PK is funded by the Wellcome Trust UK and the Oxford NIHR Biomedical Research Centre, EB is funded by the Medical Research Council UK and the Oxford NIHR Biomedical Research Centre, PK is funded by the James Martin School for 21st Century and NIH V19AI082630.

Competing interests None.

Provenance and peer review Commissioned; externally peer reviewed.

\section{REFERENCES}

1. Lechner F, Wong DK, Dunbar PR, et al. Analysis of successful immune responses in persons infected with hepatitis $C$ virus. J Exp Med 2000;191:1499-512.
2. Thimme R, Oldach D, Chang KM, et al. Determinants of viral clearance and persistence during acute hepatitis $C$ virus infection. $J$ Exp Med 2001;194:1395-406.

3. Neumann-Haefelin C, Timm J, Schmidt J, et al. The protective effect of HLA-B27 in hepatitis $C$ virus infection requires presence of a genotype-specific immunodominant CD8 + T cell epitope. Hepatology 2010;51:54-62.

4. Kenny-Walsh $\mathbf{E}$. Clinical outcomes after hepatitis $C$ infection from contaminated anti-D immune globulin. Irish Hepatology Research Group. N Engl J Med 1999;340:1228-33.

5. McHutchison JG, Lawitz EJ, Shiffman ML, et al. PEGinterferon alfa-2b or alfa-2a with ribavirin for treatment of hepatitis $\mathrm{C}$ infection. N Engl J Med 2009;361:580-93.

6. Muir AJ, Bornstein JD, Killenberg PG. PEGinterferon alfa-2b and ribavirin for the treatment of chronic hepatitis $C$ in blacks and non-Hispanic whites. N Engl J Med 2004;350:2265-71.

7. Reddy KR, Hoofnagle JH, Tong MJ, et al. Racial differences in responses to therapy with interferon in chronic hepatitis $\mathrm{C}$. Hepatology 1999;30:787-93.

8. Ge D, Fellay J, Thompson AJ, et al. Genetic variation in IL28B predicts hepatitis $C$ treatment-induced viral clearance. Nature 2009:461:399-401.

9. Rauch A, Kutalik Z, Descombes P, et al. Genetic variation in IL28B is associated with chronic hepatitis $C$ and treatment failure: a genome-wide association study. Gastroenterology 2010; 138:1338-45

10. Suppiah V, Moldovan M, Ahlenstiel G, et al. IL28B is associated with response to chronic hepatitis $C$ interferon- $\alpha$ and ribavirin therapy. Nat Genet 2009;41:1100-4.

11. Tanaka Y, Nishida N, Sugiyama M, et al. Genome-wide association of IL28B with response to pegylated interferon-[alpha] and ribavirin therapy for chronic hepatitis C. Nat Genet 2009;41:1105-9.

12. Labie D, Gilgenkrantz H. IL28 (interferon lambda3) gene polymorphisms and response to IFN-alpha treatment in patients infected with hepatitis virus C. Med Sci 2010;26:225-6.

13. Mangia A, Thompson AJ, Santoro R, et al. An IL28B polymorphism determines treatment response of hepatitis $C$ virus genotype 2 or 3 patients who do not achieve a rapid virologic response. Gastroenterology 2010:139:821-7.

14. Montes-Cano MA, Garcia-Lozano JR, Abad-Molina C, et al. Interleukin-28B genetic variants and hepatitis virus infection by different viral genotypes. Hepatology 2010:52:33-7.

15. McCarthy JJ, Li JH, Thompson A, et al. Replicated association between an IL28B gene variant and a sustained response to pegylated interferon and ribavirin. Gastroenterology 2010;138:2307-14.

16. Mosbruger TL, Duggal P, Goedert JJ, et al. Large-scale candidate gene analysis of spontaneous clearance of hepatitis $C$ virus. $\mathrm{J}$ Infect Dis 2010;201:1371-80.

17. Rallón NI, Naggie S, Benito JM, et al. Association of a single nucleotide polymorphism near the interleukin-28B gene with response to hepatitis $\mathrm{C}$ therapy in HIV/hepatitis $\mathrm{C}$ virus-coinfected patients. AIDS 2010;24:F23-9.

18. Thomas DL, Thio CL, Martin MP, et al. Genetic variation in IL28B and spontaneous clearance of hepatitis $C$ virus. Nature 2009:461:798-801

19. Cirulli ET, Goldstein DB. Uncovering the roles of rare variants in common disease through whole-genome sequencing. Nat Rev Genet 2010;11:415-25

20. Dickson SP, Wang K, Krantz I, et al. Rare variants create synthetic genome-wide associations. PLoS Biol 2010;8:e1000294.

21. Sheppard P, Kindsvogel W, Xu W, et al. IL-28, IL-29 and their class II cytokine receptor IL-28R. Nat Immunol 2003;4:63-8.

22. Kotenko SV, Gallagher G, Baurin WV, et al. IFN- $\lambda$ s mediate antiviral protection through a distinct class II cytokine receptor complex. Nat Immunol 2003;4:69-77

23. Pagliaccetti NE, Robek MD. Interferon- $\lambda$ in the immune response to hepatitis B virus and hepatitis C virus. J Interferon Cytokine Res 2010;30:585-90.

24. Coccia EM, Severa M, Giacomini E, et al. Viral infection and Tolllike receptor agonists induce a differential expression of type I and $\lambda$ interferons in human plasmacytoid and monocyte-derived dendritic cells. Eur J Immunol 2004;34:796-805.

25. Megjugorac NJ, Gallagher GE, Gallagher G. IL-4 enhances IFN- $\lambda 1$ (IL-29) production by plasmacytoid DCs via monocyte secretion of IL-1Ra. Blood 2010;115:4185-90.

26. Siren J, Pirhonen J, Julkunen I, et al. IFN- $\lambda$ regulates TLRdependent gene expression of IFN- $\alpha$, IFN- $\beta$, IL-28, and IL-29. J Immunol 2005;174:1932-7.

27. Ank N, West H, Bartholdy C, et al. Lambda interferon (IFN- $\lambda$ ), a type III IFN, is induced by viruses and IFNs and displays potent antiviral activity against select virus infections in vivo. J Virol 2006;80:4501-9. 
28. Wolk K, Witte K, Sabat R. Interleukin-28 and interleukin-29: novel regulators of skin biology. $J$ Interferon Cytokine Res 2010:30:617-28.

29. Mihm S, Frese M, Meier V, et al. Interferon type I gene expression in chronic hepatitis C. Lab Invest 2004;84:1148-59.

30. Witte K, Gruetz G, Volk HD, et al. Despite IFN- $\lambda$ recepto expression, blood immune cells, but not keratinocytes or melanocytes, have an impaired response to type III interferons: implications for therapeutic applications of these cytokines. Genes Immun 2009;10:702-14.

31. Doyle SE, Schreckhise H, Khuu-Duong K, et al. Interleukin-29 uses a type 1 interferon-like program to promote antiviral responses in human hepatocytes. Hepatology 2006;44:896-906

32. Mennechet FJD. Interferon- -treated dendritic cells specifically induce proliferation of FOXP3-expressing suppressor T cells. Blood 2006;107:4417-23.

33. Dumoutier L, Tounsi A, Michiels T, et al. Role of the interleukin (IL)-28 receptor tyrosine residues for antiviral and antiproliferative activity of IL-29/interferon-lambda 1: similarities with type I interferon signaling. J Biol Chem 2004:279:32269-74.

34. Renauld JC. Class II cytokine receptors and their ligands: key antiviral and inflammatory modulators. Nat Rev Immunol 2003;3:667-76.

35. Der SD, Zhou A, Williams BR, et al. Identification of genes differentially regulated by interferon alpha, beta, or gamma using oligonucleotide arrays. Proc Natl Acad Sci U S A 1998;95:15623-8.

36. Rani MR, Foster GR, Leung $S$, et al. Characterization of beta-R1, a gene that is selectively induced by interferon beta (IFN-beta) compared with IFN-alpha. J Biol Chem 1996;271:22878-84.

37. Gad HH, Dellgren C. Hamming OJ, et al. Interferon- $\lambda$ is functionally an interferon but structurally related to the interleukin10 family. J Biol Chem 2009:284:20869-75.

38. Marcello T, Grakoui A, Barba-Spaeth G, et al. Interferons $\alpha$ and $\lambda$ inhibit hepatitis $C$ virus replication with distinct signal transduction and gene regulation kinetics. Gastroenterology 2006;131:1887-98.

39. Dai J, Megjugorac NJ, Gallagher GE, et al. IFN- $\lambda 1$ (IL-29) inhibits GATA3 expression and suppresses Th2 responses in human naive and memory T cells. Blood 2009;113:5829-38.

40. Jordan WJ, Eskdale J, Srinivas $S$, et al. Human interferon lambda-1 (IFN- $\lambda 1 / / \mathrm{IL}-29$ ) modulates the Th1//Th2 response. Genes Immun 2007;8:254-61.

41. Srinivas S, Dai J, Eskdale J, et al. Interferon- $\lambda 1$ (interleukin-29) preferentially down-regulates interleukin-13 over other T helper type 2 cytokine responses in vitro. Immunology 2008;125:492-502

42. Morrow MP, Yan J, Pankhong P, et al. IL-28B/IFN-lambda 3 drives granzyme $\mathrm{B}$ loading and significantly increases CTL killing activity in macaques. Mol Ther 2010;18:1714-23.

43. Wolk K, Witte K, Witte $\mathrm{E}$, et al. Maturing dendritic cells are an important source of IL-29 and IL-20 that may cooperatively increase the innate immunity of keratinocytes. J Leukoc Biol 2008:83:1181-93.

44. Robek MD, Boyd BS, Chisari FV. Lambda interferon inhibits hepatitis B and C virus replication. J Virol 2005;79:3851-4.

45. Muir AJ, Shiffman ML, Zaman A, et al. Phase 1b study of pegylated interferon lambda 1 with or without ribavirin in patients with chronic genotype 1 hepatitis $\mathrm{C}$ virus infection. Hepatology 2010;52:822-32

46. Pagliaccetti NE, Eduardo R, Kleinstein SH, et al. Interleukin-29 functions cooperatively with interferon to induce antiviral gene expression and inhibit hepatitis C virus replication. J Biol Chem 2008;283:30079-89.

47. Fried MW, Shiffman ML, Reddy KR, et al. PEGinterferon alfa-2a plus ribavirin for chronic hepatitis $C$ virus infection. $N$ Engl J Med 2002;347:975-82.

48. Hadziyannis SJ, Sette H Jr, Morgan TR, et al. PEGinterferonalpha2a and ribavirin combination therapy in chronic hepatitis C: a randomized study of treatment duration and ribavirin dose. Ann Intern Med 2004;140:346-55.

49. Honda M, Sakai A, Yamashita T, et al. Hepatic ISG expression is associated with genetic variation in interleukin $28 \mathrm{~B}$ and the outcome of IFN therapy for chronic hepatitis C. Gastroenterology 2010; 139:499-509.

50. Sarasin-Filipowicz M, Oakeley EJ, Duong FHT, et al. Interferon signaling and treatment outcome in chronic hepatitis C. Proc Natl Acad Sci U S A 2008;105:7034.

51. Chen L, Borozan I, Sun J, et al. Cell-type specific gene expression signature in liver underlies response to interferon therapy in chronic hepatitis C infection. Gastroenterology 2010;138:1123-33.

52. Muir AJ, Lawitz E, Ghalib RH, et al. Pegylated interferon lambda (pegIFN $\lambda$ ) phase 2 dose-ranging, active-controlled study in combination with ribavirin (RBV) for treatment-naïve HCV patients (genotypes 1, 2, 3 or 4): safety, viral response, and impact of IL28B host genotype through week 12. Hepatology 2010:52:715A-16A.

53. Contoli M, Message SD, Laza-Stanca V, et al. Role of deficient type III interferon- $\lambda$ production in asthma exacerbations. Nat Med 2006;12:1023-6. 